\title{
Design synthesis and performance: simulation, discussion and generative strategies
}

\section{SIGRADI2018 TECHNOPOLITICAS \\ xxii congresso da sociedade iberoamericana de gráfica digital 22th conference of the iberoamerican society of digital graphics 07|08|09|novembro|2018 iau usp | são carlos | sp br}

\author{
Thiago Silva \\ Universidade Federal de Uberlândia | Brazil | thigo@ufu.br \\ Juliana Lima \\ Universidade Federal de Uberlândia | Brazil | juhmonteiro@ufu.br \\ Nicole Maia \\ Universidade Federal de Uberlândia | Brazil | nicole.maia@ufu.br \\ André Araujo \\ Universidade Federal de Uberlândia | Brazil | andre.araujo@ufu.br
}

\begin{abstract}
Since the publication of the book Performance architecture: beyond instrumentality (KOLAREVIC and MALKAWI,2004), architects have used a good performance as a guideline for the projects. This research proposes to investigate the possibilities and limitations of one of the guidelines through the incorporation of instantaneous aerodynamics in the parametric design context, from the use of parametric modeling techniques, electronic microcontrollers and computational extensions to promote the connection between them. From the construction of this artifact, we expect to develop strategies for including performance simulations in the processes of synthesis in architecture.
\end{abstract}

Keywords: Ventilation; Wind-sensors; Parametric Design, Arduino.

\section{INTRODUÇÃO}

Embora se possa considerar a revolução digital do início dos anos 2000 como um marco fundamental para a pesquisa em Design Computacional, devido à introdução contundente das linguagens de programação nos processos de modelagem, o estreitamento da relação entre a Arquitetura e a Tecnologia da Informação é bem anterior a isso. No início do século anterior, os primeiros experimentos que conduziram ao que se entende hoje por estruturas espaciais trianguladas ou treliças, utilizadas com frequência em coberturas ou pórticos de edifícios, foram desenvolvidas por Alexander Graham Bell, que em 1906 fundou a Aerial Experiment Association, uma organização destinada a construir kits de torres pré-fabricadas (SHANNON, 1948). Neste momento da história, a figura do inventor do telefone parece ser um elo entre estas duas áreas de conhecimento, permitindo-o estabelecer funções e objetivos na investigação de formas a partir de módulos geométricos de octaedros e tetraedros, a fim de construir torres de telefonia.

Ao produzir suas estruturas espaciais, Bell provavelmente não imaginava que um século mais tarde elas seriam projetadas utilizando a mesma tecnologia digital de fluxo de informação presente nos telefones (ARAUJO, 2017; 2018). A partir dos anos 1960, a indústria automobilística e aeroespacial passou a utilizar os computadores como uma ferramenta de auxílio aos processos de projeto (CAD)1 e, pouco tempo depois, estas tecnologias adentraram também os escritórios de arquitetura e engenharia (SHUTERLAND, 1963). A partir dessa época, duas práticas fundamentais se consolidaram nos processos de design, em suas diferentes instâncias (GERO, 1996): 1) a representação da geometria de objetos acompanhada da automatização de tarefas repetitivas; 2) o uso da computação nos processos de síntese do design. Enquanto o primeiro se popularizou nos escritórios, por meio de aplicativos comerciais, o último se restringiu a experimentações e aplicações específicas.

Já nos anos 1980, os arquitetos Peter Eisenman e Greg Lynn sugeriram o uso de algoritmos em detrimento das formas clássicas de desenho técnico para a definição do espaço arquitetônico. De acordo com Eisenman (1996), a utilização da projeção planimétrica na arquitetura representava uma visão problemática, principalmente por obrigar o entendimento do espaço tridimensional em duas dimensões2. A proposta de uma arquitetura algorítmica foi, em alguma medida, um reflexo das transições das plataformas mecânica para eletrônica, do mesmo período. Além das mudanças nas noções cartesianas, a noção algorítmica viria a ser apropriada por arquitetos contemporâneos nos anos subsequentes, que desenvolveram regras, raciocínios e operações matemáticas aplicadas a um número finito de dados, a fim de solucionar problemas do espaço (BURRY e BURRY, 2012).

Nos anos 1990, os esforços de alguns arquitetos em construir formas complexas culminaram no desenvolvimento de técnicas de modelagem algorítmica ou paramétrica (TERZIDIS, 2006). Celani (2012) demonstrou este ponto como uma inflexão na cultura do 
CAD, que são as novas relações dos processos de modelagem com as linguagens de programação a partir da proposta de uma representação visual dos scripts, que passaram a ser incluídos nos aplicativos CAD como scripts visuais, com porções de código incorporado a cápsulas gráficas (BARBOSA et al., 2012). Provavelmente, o exemplo mais contundente dessa prática são os métodos de projeto introduzidos pelo arquiteto canadense Frank Gehry que, em plataformas digitais de projeto, vinculou os processos de modelagem e fabricação permitindo a investigação de formas, a partir de parâmetros produtivos incorporados durante 0 processo criativo dentro do aplicativo Digital Project (Gehry Technologies, Inc., 2018). Em um estudo aprofundado dos métodos de projeto de Gehry, Shelden (2002) concluiu que o processo permite avaliações críticas a todo o momento, permitindo a construção de uma estrutura formal descrita de maneira inequívoca por um protótipo físico, mas também passível de ser alterada pela inserção de novos dados de projeto.

A partir dos 2000, os scripts visuais foram incorporados por outros aplicativos de modelagem utilizados pelos arquitetos, como o Generative Components (BENTLEY, Inc., 2018) e o Rhinoceros 3D (McNEEL, Inc., 2018), o que simplificou a incorporação das linguagens de programação nos estágios criativos de projeto. Com efeito, diversas variáveis objetivas puderam ser facilmente computadas em um modelo virtual, por meio de relações matemáticas que permitem a alteração do elemento geométrico a cada inserção de novo dado, da mesma maneira que uma planilha recalcula automaticamente alterações numéricas (KOLAREVIC, 2005). O arquiteto Norman Foster foi um dos pioneiros a explorar esse raciocínio ao propor a Torre Suiça, em Londres. Neste projeto, um modelo virtual paramétrico foi produzido visando a incorporação de análises aerodinâmicas durantes o processo criativo. Desse modo, a forma da estrutura diagonal utilizada na fachada do edifício poderia ser investigada com base em avaliações de vento instantâneas e, assim, produziam-se diversos candidatos à solução de projeto em detrimento de uma solução única. O resultado foi uma torre baseada na espiral, cujo raio aumenta proporcionalmente com a altura e, em seguida, se afunila para 0 vértice (FOSTER+PARTNERS, Inc., 2018).

Desde a publicação da obra Performative Architecture: Beyond Instrumentality (KOLAREVIC e MALKAWI, 2005), os arquitetos têm se preocupado cada vez mais em utilizar o bom desempenho como um princípio norteador do projeto. Essa conduta sempre esteve presente na obra de arquitetos como Antoni Gaudí, Henz Isler e Frei Otto, mas, segundo esses autores, a disponibilidade atual de programas de simulação facilitou enormemente a incorporação dos resultados das análises de desempenho, em especial, o ambiental e o estrutural, nas etapas iniciais de projeto. No entanto, a retroalimentação dos resultados dessas análises nem sempre é feita de maneira automatizada, geralmente exigem técnicas de modelagem paramétrica e de prototipagem rápida, o que implica necessariamente na adoção de condutas projetuais específicas.

Esta pesquisa pretende avaliar uma destas condutas e tem como questão central o delineamento e a incorporação da performance como um novo paradigma o processo criativo em arquitetura. A partir de desenvolvimentos recentes que possibilitaram a inserção das linguagens de programação e da prototipagem eletrônica nas plataformas digitais de projeto, pretende-se explorar instrumentos e métodos destinados a propor novas maneiras de entendimento sobre como os edifícios podem ser imaginados, construídos e experienciados. A partir de um modelo de simulações aerodinâmicas urbanas desenvolvido por Prohasky et al. (2012), que investigaram a anemometria a partir de protótipos eletrônicos de código aberto, pretende-se explorar uma nova abordagem para os arquitetos baseada na influência do feedback em tempo real do vento quantificado na compreensão dos fenômenos eólicos, estimulando a sua compreensão intuitiva.

\section{MÉTODO}

Esta pesquisa tem um marco teórico exploratório, no qual se busca a aproximação a particularidades das condutas projetuais contemporâneas, a fim de tornar essa atividade mais explícita e construir hipóteses sobre seus processos. A partir um estudo bibliográfico sobre os temas desta pesquisa, propõe-se construção de um artefato tecnológico com base nas método incremental e iterativo, tradicionalmente utilizado na Engenharia de Software, que pressupõe a construção algorítmica a partir da programação de uma funcionalidade central e de incrementos posteriores. Estes procedimentos empregarão técnicas de modelagem paramétrica prototipagem eletrônica, conforme os resultados obtidos em um pré-teste.

Utilizando um arquivo climático obtido na base de dados produzida por Roriz (2018), nos formatos Energyplus Weather Data (Energyplus, Inc., 2018), realizaram-se testes com os aplicativos gráficos Rhinoceros 3D v. 5 (McNell, Inc., 2018), e a extensão de edição gráfica de scripts Grasshopper 3D v.0.90076 em conexão com a plataforma de prototipagem eletrônica de código aberto Arduino (Arduino, Inc., 2018). Do arquivo climático foram obtidos os registros de ventilação com direções predominantes e quantidades de horas por direção ao longo das 8.760 horas do ano. À placa de prototipagem eletrônica foram conectados a placa controladora Arduino NANO, o Hotwire anemometer modern device (revC) e um display digital, que também pode ser visualizado em tela. Por meio das subextensões Ladybug (leitura do output do arquivo climático no Rhinoceros 3D) e Firefly (leitura do output do Arduino no Rhinoceros 3D) desenvolveram-se algoritmos para a visualização gráfica dos comportamentos de vento em nível global e local, equacionando os registros anuais e instantâneos.

Por meio de procedimentos não necessariamente sequenciais, organizados de maneira cíclica e com sobreposições, define-se um marco conclusivo (exercício projetual), onde pretende-se estabelecer comparativos a partir da inserção do artefato em processos de tradicionais de projeto: a. Requisitos, análise e planejamento: revisão de literatura e procedimentos de engenharia reversa, estudos de caso, elaboração de um programa de necessidades e esboços preliminares do artefato b. Implementação e desenvolvimento: prescrições concretas de soluções computacionais, desenvolvidas a partir de conclusões empíricas obtidas nos outros dois grupos. c. Avaliações, testes e discussão: 
procedimentos de exequibilidade do artefato proposto, avaliações comparativas com protótipos virtuais e físicos e um exercício projetual.

Com base neste recorte analítico proposto, que considera apenas as variáveis climáticas de ventilação, pretende-se discutir e propor hipótese para a aplicação dos conceitos utilizados em outros tipos de análise de performance.

\section{RESULTADO EXPLORATÓRIO}

Com base em um modelo de simulações aerodinâmicas urbanas desenvolvido por Prohasky et al. (2012) e Wlliams et al. (2015), que investigaram a anemometria a partir de protótipos eletrônicos de código aberto, buscouse investigar possibilidades para a vinculação das técnicas de design paramétrico e de prototipagem eletrônica.

\section{DESIGN PARAMÉTRICO}

O modelo paramétrico proposto consistiu de um abrigo cujas projeções horizontais constituem um quadrado de $30 \times 30$ metros e foi obtido a partir da modelagem de uma superfície gerada por curvas-base do tipo B-Splines, modeladas no aplicativo Rhinoceros (McNell, Inc.). Os pontos de controle das curvas foram discretizados a partir de suas coordenadas $\mathrm{X}, \mathrm{Y}$ e $\mathrm{Z}$, as quais podem ser livremente alteradas modificando-se, por conseguinte, a geometria da superfície. À esta superfície vinculou-se uma sequência algorítmica desenvolvida no editor de scripts visuais Grasshopper (McNell, Inc.), a fim de gerar uma estrutura portante composta por dois conjuntos ortogonais de placas verticais do tipo waffle, conectadas entre si por meio de encaixes similares aos de uma caixade-ovo (egg crate). Desse modo, a cada mudança de coordenada $X Y Z$ dos pontos de controle das B-Splines, a superfície vinculada também muda e, em consequência, a sua estrutura portante. A partir desse modelo paramétrico, determinou-se uma instância para essa estrutura portante com base em dados climáticos sobre os registros de vento na cidade $X$, obtidos no arquivo climático produzido por Roriz (2018). Tendo como base o elemento instanciado, produziu-se um protótipo na escala 1:50 utilizando equipamentos cnc de corte a laser (Fig. $1 \mathrm{a}-\mathrm{c})$.
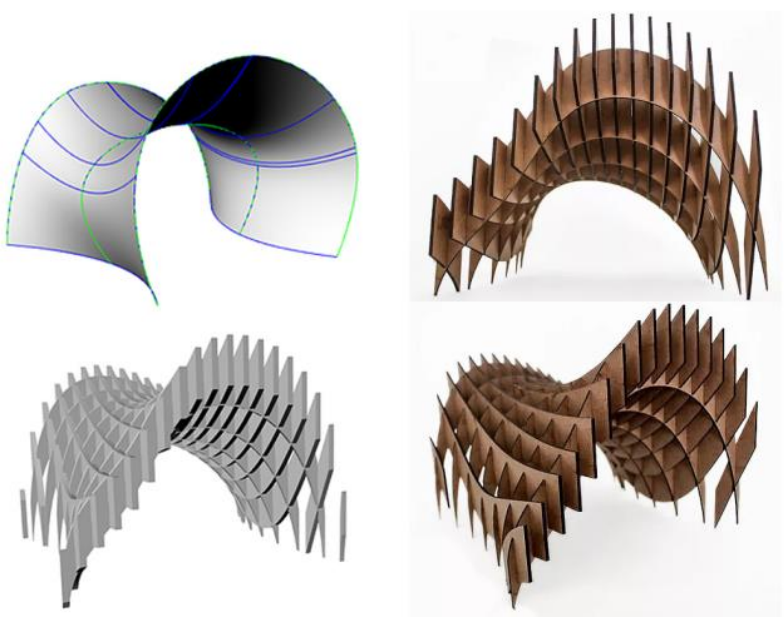

Figura 1: Design paramétrico e prototipagem de uma instância da estrutura portante. Fonte: Autores.

\section{PROTOTIPAGEM ELETRÔNICA}

Por meio de um protótipo eletrônico construído na plataforma de código aberto Arduino, propôs-se a detecção de vento como um método para a observação fenomenológica da dinâmica de vento pela quantificação de experimentos empíricos em tempo real. Utilizando a extensão Firefly, capaz de conectar as respostas do Arduino ao Grasshopper (McNell, Inc.) obteve-se um feedback das mudanças no vento em unidades compreensíveis $(\mathrm{m} / \mathrm{s})$ em pontos estratégicos em torno do abrigo. A cada ponto de ensaio vinculou-se uma esfera de parâmetro variável, cujo raio se altera conforme os registros de vento do anemômetro. Desse modo, a plataforma sensora integra três principais tecnologias nesta abordagem: simulações físicas de vento com um túnel de vento, sensores micro anemômetros conectados a uma placa Arduino e uma interface digital para visualizar dados usando Grasshopper (0.09.0056) com o plugin Firefly e o aplicatvo Rhino5.0.
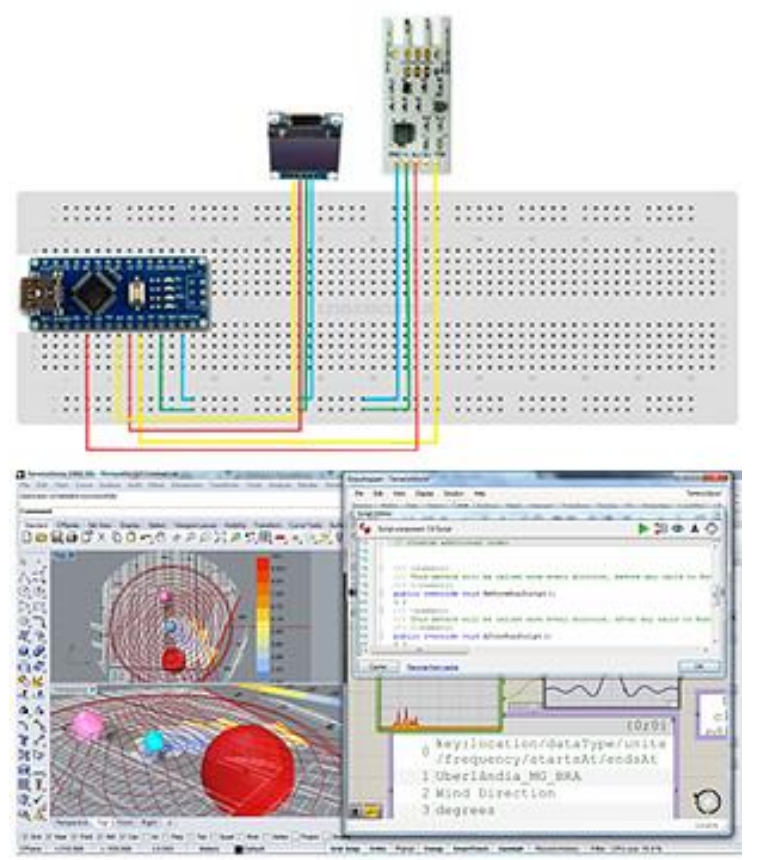

3

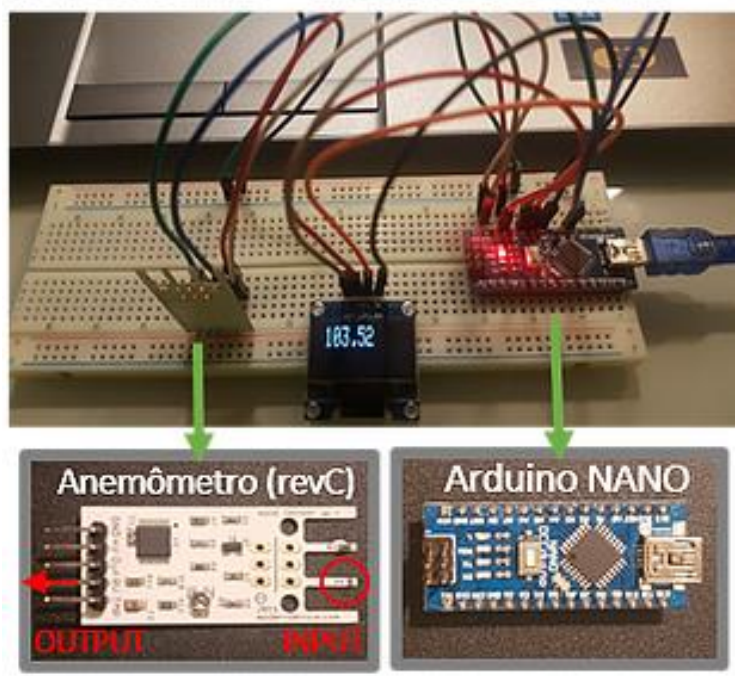

Figura 2: Prototipagem eletrônica e sua vinculação com os aplicativos Rhinoceros e Grasshopper, promovendo feedbacks instantâneos em tempo-real. Fonte: Autores. 


\section{RESULTADO EXPERIMENTAL}

Os experimentos foram realizados no túnel de vento do laboratório Y que possui abertura quadrada de $60 \times 60$ $\mathrm{cm}^{2}$. A dinâmica do fluxo é criada por um rotor de 12 lâminas impulsionado por um $25 \mathrm{Hp}$ motor elétrico. A velocidade máxima do ar no túnel seção de teste é de aproximadamente $30 \mathrm{~m} / \mathrm{s}$ e, para este ensaio, foi utilizado velocidades entre 2 e $15 \mathrm{~m} / \mathrm{s}$, que são as velocidades de vento usuais nas condições de serviço das edificações.

A fim de visualizar o fluxo e a separação/recirculação de vento das zonas próximas à superfície do modelo, no modelo virtual, aplicou-se uma escala cromática gradativa do vermelho ao violeta à superfície das esferas, vinculando o seu raio às cores, ou seja, quanto maior o fluxo de vento registrado, maior o raio da esfera e, consequentemente, mais próxima é a sua cor do ultravioleta. Comparando os registros em diferentes esferas, duas características importantes do fluxo perto da superfície puderam ser facilmente apontadas: direção laminar dos fluxos e as regiões turbulentas ou instáveis.
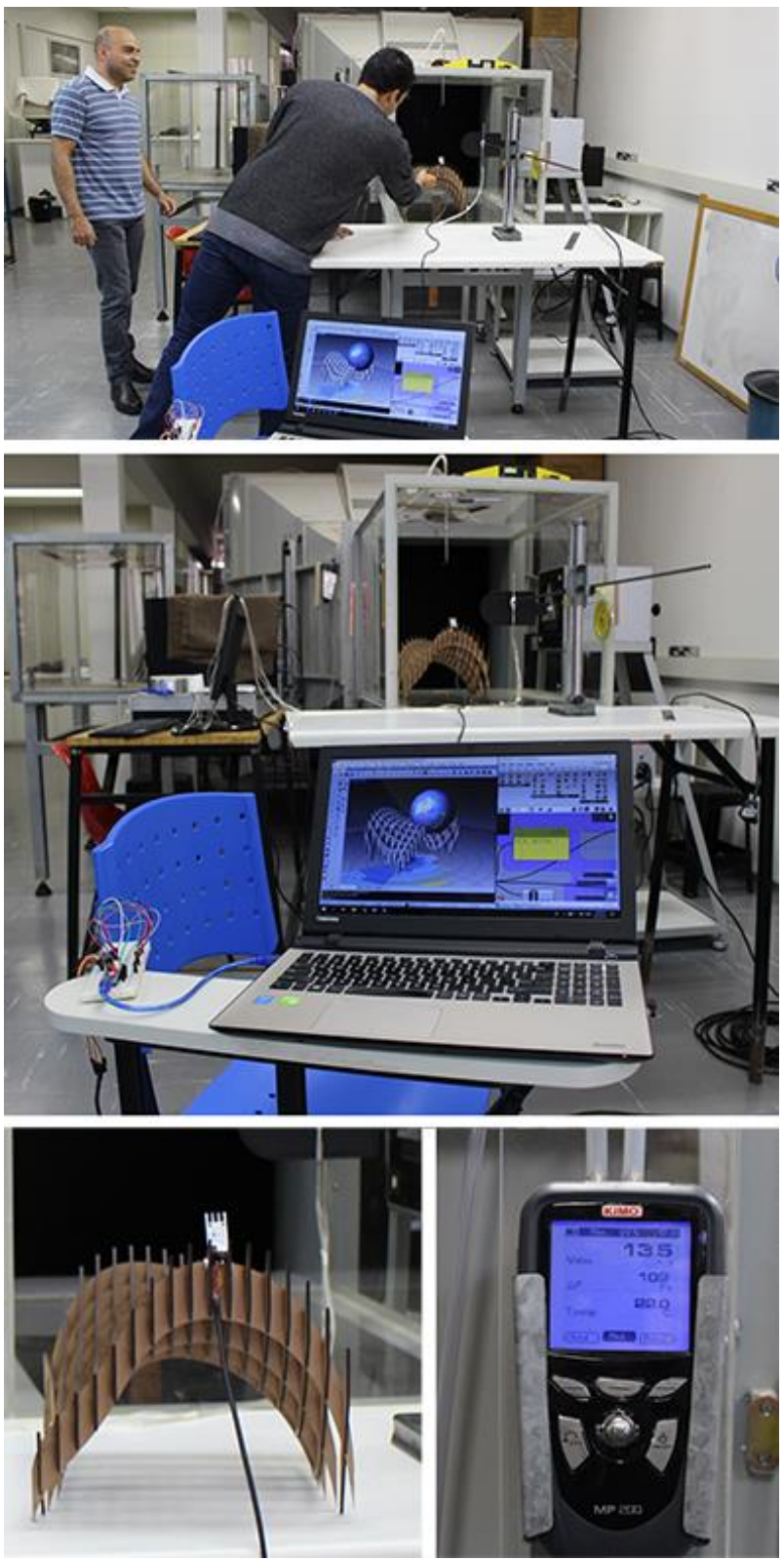

Figura 3: Ensaio realizado em túnel de vento. Fonte: Autores.
Os sensores foram estrategicamente colocados em pontos específicos do protótipo e, assim, foi possível identificar acelerações provocadas pela própria configuração formal do elemento. Esses efeitos foram reconhecidos pelas assimetrias existentes entre as esferas e estas se tornaram muito úteis para a análise do processo. Essas observações foram registradas por meio de uma de gravação de vídeo e áudio durante o experimento. Uma seleção dessas gravações será apresentada na conferência.

\section{DISCUSSÃO}

Em geral, o tratamento dos problemas de ventilação natural nos projetos das áreas de Arquitetura, Engenharia e Construção (AEC) são feitos utilizando informações sobre a macro ventilação com direções predominantes de vento e quantidades de horas por direção ao longo das 8.760 horas do ano. Na macroescala, questões relativas à micro ventilação geralmente são delineadas por meio de dispositivos eletrônicos, como os anemômetros, cuja precisão é satisfatória para a maioria dos problemas da área. Em grande parte dos problemas de projeto, ambas as análises funcionam como um procedimento a posteriori, isto é, como uma validação às formas definidas durante os estágios criativos. Nos últimos anos, alguns projetos de edifícios altos como o The Swiss Tower, em Londres, de autoria do escritório britânico Foster+Partners, se tornaram ícones da arquitetura contemporânea ao incluir a performance como variável norteadora de projeto, neste caso, vinculando o registro das pressões de vento a um sistema de parâmetros variáveis capaz de se atualizar a forma do edifício com base nos valores registrados. Desse modo, a estrutura do edifício poderia ser investigada com base em avaliações de vento instantâneas e, assim, produziam-se diversos candidatos à solução de projeto.

O uso de métodos de simulação do microclima, em geral, permite a análise de variáveis diversas e, consequentemente, a produção de cenários capazes de informar os processos decisórios em arquitetura. Diferentemente das variáveis climáticas estáticas, como a trajetória solar, a ventilação em ambientes urbanos se encontra entre as variáveis de mais difícil delineamento em virtude de sua instabilidade.

Nas condições do ensaio, as velocidades médias registradas nos pontos da estrutura apresentam velocidades semelhantes e, exatamente por isso, a configuração da precisão do anemômetro RevC precisa expressar pelos menos três casas decimais. Contudo, as técnicas de modelagem paramétrica utilizadas nos raios das esferas permitiram majorar inclusive as pequenas diferenças de ventilação. Nesses casos, as questões de escala do elemento (1:50) e da intrusão do próprio anemômetro na análise (as dimensões do RevC na escala 1:50 representam uma barreira de aproximadamente 2 metros) devem ser consideradas. Os diferenciais entre os pontos registrados são apresentados na Figura 4. 

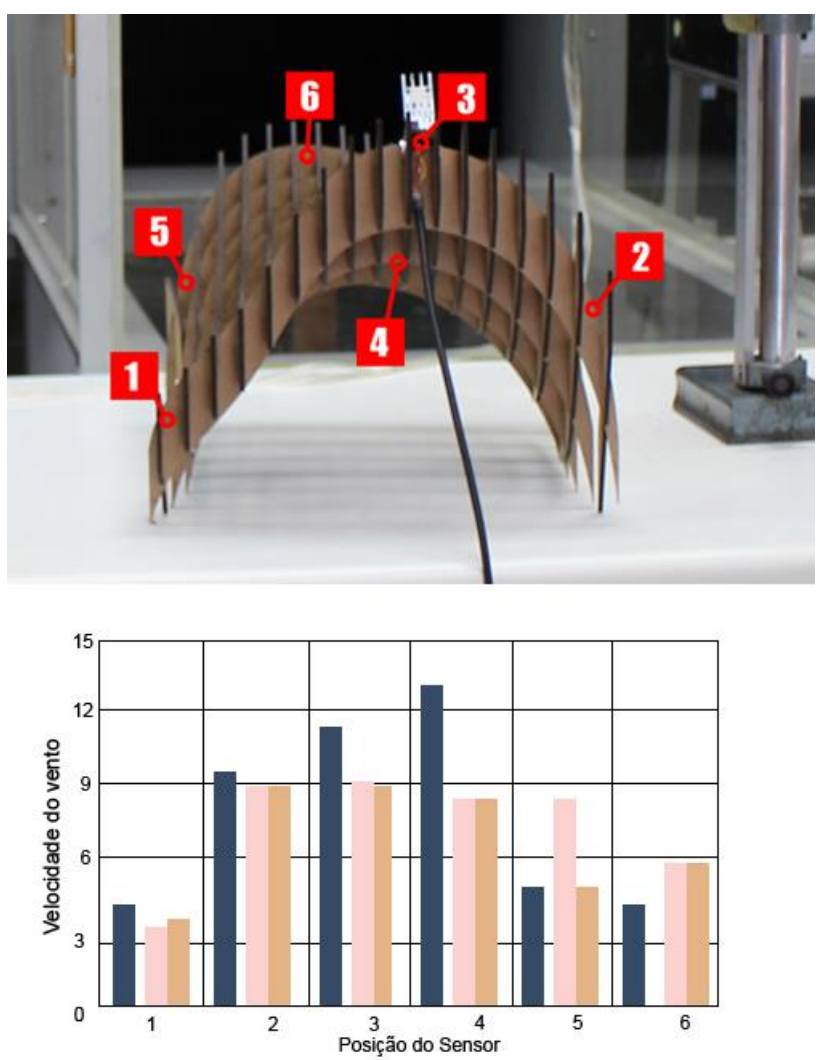

Figura 4: Resultados obtidos nos diferentes pontos de análise da forma. Fonte: Autores.

As observações empíricas sobre a dinâmica do vento são, provavelmente, as maneiras mais eficazes e informativas para se entender este comportamento nas superfícies, tanto do abrigo experimental em questão, quanto em fachadas de edifícios. Desse modo, é possível que os métodos de projeto insiram a variável vento durante os processos de projeto, mesmo sem um grande conhecimento sobre a mecânica dos fluidos, a partir do entendimento empírico sobre os fenômenos aerodinâmicos nos ambientes construídos. Este tipo de detecção, abre uma oportunidade de experimentar soluções em tempo-real e consequentemente, orientar uma tomada de decisão. A partir da união desses dois conceitos expressos em artefatos físicos e virtuais, que permitem uma alteração rápida da forma a partir de um modelo paramétrico e de um feedback rápido sobre a dinâmica de vento, algumas estratégias de modelagem se tornam eficazes:

a. Superfícies que possam ser controladas por pontos são mais bem aproveitadas neste tipo de análise, principalmente se os sensores forem posicionados em seus pontos de controle.

b. Estruturas de placas como as definições de waffle utilizadas são mais adequadas, por permitirem um ajuste de aberturas, aumentando ou diminuindo o número de placas.

c. Percebeu-se uma certa incompatibilidade entre as leituras das portas lógicas do Arduino e do Firefly, de modo que um dos dois deve estar desligado para que o outro funcione.

\section{CONCLUSÃO}

Embora os resultados desta pesquisa sejam ainda preliminares, é possível concluir que a aplicação da plataforma do sensor para visualização e compreensão de simulações físicas pode melhorar a observação e análise de dinâmica do vento no ambiente construído para arquitetos e designers, potencializando a comunicação com outros especialistas. Esta ferramenta tem o potencial para ser usado no estudo e projeto de microclima, criando condições para áreas de pedestre próximas a edifícios.

Portanto, esta pesquisa apresentou uma plataforma tecnológica que integrou diferentes técnicas de simulação e visualização de fenômenos eólicos para a análise de simulações aerodinâmicas do fluxo de vento nas edificações. Essa tecnologia tem o potencial de auxiliar arquitetos e designer com poucos conhecimentos sobre a mecânica dos fluidos a entender melhor a dinâmica de vento em torno dos edifícios. Possivelmente, esta é uma ferramenta prática para analisar problemas de conforto produzidos pelo vento em áreas próximas a edifícios, mas também, é uma ferramenta que pode ajudar os profissionais a explorar a aerodinâmica dos ambientes através de observações empíricas e quantificáveis. Além disso, é uma tecnologia de custo muito baixo, confiável que pode ser facilmente utilizada nos escritórios nos projetos em que os efeitos de vento forem relevantes.

\section{AGRADECIMENTOS}

Os autores agradecem à concessão APQ 01926-17 da Fundação de Amparo à Pesquisa do Estado de Minas Gerais e ao Conselho Nacional de Desenvolvimento Científico e Tecnológico pela bolsa de Thiago Silva.

\section{REFERÊNCIAS}

Araujo, A. (2018). Autômatos celulares: definição e aplicações na arquitetura. In G. Celani, M. Sedrez (Eds.), Arquitetura contemporânea e automação: prática e reflexão (pp. 69-84). São Paulo, SP: Probooks.

Araujo, A.; Celani, G. (2017). Interpretações arquitetônicas dos autômatos celulares: conceitos e aplicações recentes. Cadernos Proarq, 29(2), 154-174.

Barbosa, W., Araujo, A., Carvalho, G., Celani, G. (2012). Samba Reception Desk: compromising aesthetics, fabrication and structural performance in the design process. International Conference on Education and Research in Computer-Aided Architectural Design in Europe, 30, 245-254. Retrieved from http://papers.cumincad.org/cgi-

bin/works/Show?ecaade2012 163

Bentley INC. In: Bentley. (2018). Retrieved from http://www.bentley.com/

EnergypluS, INC. In: Energyplus. (2018). Retrieved from https://energyplus.net/.

Burry, J.; Burry, M. (2012).The New Mathematics of Architecture. Londres: Thames \& Hudson.

Eisenman, P. (2nd ed.)(1996) Visions unfolding: architecture in the age of eletronic media. In: Nesbitt, K. Theorizing a New Agenda for Architecture, (pp.556-561). Nova Jersey: Princeton Architectural Press.

FOSTER+PARTNERS, INC. In: FOSTER + PARTNERS: Architectural design and engineering firm. (2018). Retrieved from https://www.fosterandpartners.com.

GEHRY TECHNOLOGIES. In: Gehry Technologies. (2014). Retrieved from http://www.gehrytechnologies.com/.

Gero, J. (1996) Closing discussion. In: GERO, J. Advances in Formal Design Methods for CAD, 291-298. Londres: Chapman \& Hall. 
Kolarevic, B.(2005). Architecture in the digital age. Nova lorque: Spon Press.

Kolarevic, B.; Malkawi, A. M. (Ed.).(2004). Performative architecture: beyond Instrumentality. Nova lorque: Spon Press.

Mcneel, INC. (2018). In: Robert McNeel \& Associates. Retrieved from http://www.mcneel.com/.

Prohasky, D.; Moya, R.; Watkins, S.; Burry, J.; Burry, M. (2012). Wind sensing with real-time visualisations for designers. In:

International Conference On Education And Research In Computer Aided Architectural Design In Europe, 32, Newcastle upon Tyne. 165-171.

Shannon, C. E.(1948). A mathematical theory of communication. The Bell System Technical Journal, 27(3), 379-423.
Shelden, D. R.(2002). Digital Surface Representation and the Constructability of Gehry's Architecture. 340 f. Tese (Doutorado em Arquitetura, Design e Computação)Massachusetts Institute of Technology, Boston.

Terzidis, K.(2006). Algorithmic Architecture. Oxford: Architectural Press.

Williams, M.; Moya, R.; Prohasky, D.; Latifi, M.; Watkins, S.; Burry, M.; Burry, J.; Belesky, P. (2015). A physical and numericalsimulation strategy to understand the impact of the dynamics in air for the design of porous screens. In: SIMAUD Conference, 6 . Washington.

RORIZ ENGENHARIA BIOCLIMÁTICA S/S LTDA. Roriz Bioclimática: conforto térmico e eficiência energética. (2018). Retrieved from http://www.roriz.eng.br/epw_9.html 